\title{
A study of the kinetics and mechanism of the adsorption and anaerobic partial oxidation of n-butane over a vanadyl pyrophosphate catalyst
}

\begin{abstract}
The interaction of n-butane with a ((VO)2P2O7) catalyst has been investigated by temperature-programmed desorption and anaerobic temperature-programmed reaction. nButane has been shown to adsorb on the (VO)2P2O7 to as a butylï hydroxyl pair. When adsorption is carried out at $223 \mathrm{~K}$, upon temperature programming some of the butylï hydroxyl species recombine resulting in butane desorption at $260 \mathrm{~K}$. However, when adsorption is carried out at $423 \mathrm{~K}$, the hydroxyl species of the butylï hydroxyl pair migrate away from the butyl species during the adsorption, forming water which is detected in the gas phase. Butane therefore is not observed to desorb at $260 \mathrm{~K}$ after we lowered the temperature to $223 \mathrm{~K}$ under the butane/helium from the adsorption temperature of $423 \mathrm{~K}$ prior to temperature programming from that temperature to $1100 \mathrm{~K}$ under a helium stream. Anaerobic temperature-programmed oxidation of n-butane produces butene and butadiene at a peak maximum temperature of $1000 \mathrm{~K}$; this is exactly the temperature at which, upon temperature programming, oxygen evolves from the lattice and desorbs as O2. This, and the fact that the amount of oxygen desorbing from the (VO)2P2O7 at $1000 \mathrm{~K}$ is the same as that required for the oxidation of the n-butane to butene and butadiene, strongly suggests (i) that lattice oxygen as it emerges at the surface is the selective oxidant and (ii) that its appearance at the surface is the rate-determining step in the selective oxidation of n-butane. The surface of the (VO)2P2O7 catalyst on which this selective oxidation takes place has had approximately two monolayers of oxygen removed from it by unselective oxidation of the n-butane to $\mathrm{CO}, \mathrm{CO}$, and $\mathrm{H} 2 \mathrm{O}$ between 550 and $950 \mathrm{~K}$ and has had approximately one monolayer of carbon deposited on it at $1000 \mathrm{~K}$. It is apparent, therefore, that the original crystallography of the (VO)2P2O7 catalyst will not exist during this selective oxidation and that theories that relate selectivity in partial oxidation to the (100) face of the (VO)2P2O7 catalyst cannot apply in this case.
\end{abstract}

Keyword: Partial oxidation; Vanadyl pyrophosphate catalyst; n-butane; Kinetics; Mechanism; Adsorption 